\title{
ENERGY PROPAGATION IN DISSIPATIVE SYSTEMS \\ Part I: Centrovelocity for linear systems
}

\author{
Embrecht VAN GROESEN \\ Department of Applied Mathematics, University of Twente, NL-7500 AE Enschede, The Netherlands \\ Francesco MAINARDI \\ Department of Physics, University of Bologna, I-40126 Bologna, Italy
}

Received 1 October 1987, Revised 28 April 1988

\begin{abstract}
Based on a balance equation for the energy density, an expression for the centrovelocity is derived which differs from the usual energy-flux velocity by a loss-flux term as a consequence of the presence of dissipation. In this part the expression is investigated for linear wave equations, in particular for first-order hyperbolic systems.
\end{abstract}

\section{Introduction}

In this paper we deal with the almost classical problem of the energy propagation associated with a given dispersive wave motion.

Usually, in the literature the energy-flux velocity is assumed to specify this propagation, and it is known simply as energy velocity. For homogeneous monochromatic waves propagating in a linear isotropic system with dispersion but without dissipation, this velocity coincides with the group velocity as is well known; the statement can be proved in different ways (see, e.g., [1-8]).

In order to be able to treat energy propagation for nonperiodic waves with finite energy and to incorporate dissipative effects as well, the concept of centrovelocity of energy (or, simply centrovelocity) has proved to be useful (see, e.g., [9-12]).

The centrovelocity, defined as the velocity of the center of gravity of some density, here the energy density, has a clear physical interpretation. Van Groesen [12] has shown that, for linear dispersive waves governed by a scalar and conservative wave equation, this velocity can be related to an appropriate average of the classical group velocity.
Furthermore, it was shown there that this concept can also be applied to nonlinear wave equations such as Korteweg-de Vries (KdV) and BenjaminBona-Mahony (BBM) equations.

In this paper we will restrict the analysis to uniaxial waves. Starting from a given balance equation, we recall in Section 2 the concepts of energy-flux velocity (denoted by $V_{\mathbf{r}}$ ) and of centrovelocity (denoted by $V_{\mathrm{e}}$ ). In Section 3 a quite general expression for the centrovelocity is derived. It is shown that this velocity differs from the energy-flux velocity by some term that is due to the presence of dissipation. For a class of solutions, which we refer to as uniformly-damped solutions, we prove the coincidence of $V_{\mathrm{e}}$ with $V_{\mathrm{f}}$. Only in such a case will we use the common term "energy velocity" to denote the two velocities.

After this general part we restrict our attention to linear wave equations. In Section 4 we derive, for a linear equation of first-order in time,

$$
\partial_{t} u+L u=0
$$

the expression of the centrovelocity for a given solution $u=u(x, t)$ in terms (of the symmetric and antisymmetric part) of the operator $L$; the particular case of hyperbolic systems is considered. 
In the Appendix we consider two simple linear evolution equations of physical interest in order to illustrate some aspects of our theory.

\section{The energy balance}

In order to investigate energy propagation by a given wave motion, a balance equation for the energy density is required. For uniaxial propagation in the $x$-direction conservation of energy, as is well known, is described by an equation of the form

$$
\partial_{1} \mathscr{E}+\partial_{x} \mathscr{F}=0,
$$

for all $x$ and $t$, where $\mathscr{E}=\mathscr{E}[\boldsymbol{u}]$ is the energy density of the system under consideration, $\mathscr{F}=\mathscr{F}[\boldsymbol{u}]$ is the flux density corresponding to $\mathscr{E}$, and $\boldsymbol{u}=\boldsymbol{u}(x, t)$ is the field variable that satisfies a (uniaxial) wave equation.

In that case the system is called conservative (with respect to $\mathscr{E}$ ), since integration over $x$ of (2.1) leads to the conservation of the integral $\left\{\int \mathscr{E}[\boldsymbol{u}(x, t)] \mathrm{d} x\right\}$, for any solution $\boldsymbol{u}$ satisfying suitable boundary conditions at the end of the integration interval (e.g., descent behaviour at infinity, or periodicity). Then we write

$$
\partial_{t}\langle\mathscr{E}[\boldsymbol{u}]\rangle=0,
$$

where by $\langle\cdot\rangle$ we mean integration over all of $\mathbb{R}$, or over the period. The functional $\langle\mathscr{E}[u]\rangle$ is then said to be an invariant integral of the system, and $\mathscr{E}[\boldsymbol{u}]$ is referred to as a conserved density.

For a nonconservative system, for which $\langle\mathscr{E}\rangle$ is no longer an invariant integral, one considers the balance law for $\mathscr{E}$. The usual way is to write

$$
\partial_{t} \mathscr{E}+\partial_{x} \mathscr{F}=-\mathscr{Y}
$$

In this expression we note that $\mathscr{F}=\mathscr{F}[\boldsymbol{u}]$ and $\mathscr{S}=$ $\mathscr{S}[\boldsymbol{u}]$ are not uniquely determined. Indeed, if for a given $\mathscr{E}$ the pair $\{\mathscr{F}, \mathscr{S}\}$ satisfies (2.3), then so does the pair $\left\{\mathscr{F}+\Psi, \mathscr{S}-\partial_{x} \Psi\right\}$ for any arbitrary density $\Psi$. We will call $\mathscr{S}$ the loss density if $\mathscr{F}$ is the flux of the conservative part of the system (as in $(2.1)$ ).
Let us now specify, with respect to the space variable $x$, the class of solutions $\boldsymbol{u}=\boldsymbol{u}(x, t)$ that will be considered, i.e., finite-energy solutions.

By finite-energy solutions we mean squareintegrable solutions that are defined on all of $\mathbb{R}$ and that decay, together with their derivatives, sufficiently fast at infinity to guarantee that all the densities that are relevant, and their first moments, are integrable.

For a density $\mathscr{D}[u]$ corresponding to such solutions, we denote

$$
\langle\mathscr{D}\rangle=\int_{-x}^{+\infty} \mathscr{D}[u(x, t)] \mathrm{d} x .
$$

Since we only consider densities that are normalized like $\mathscr{D}[\boldsymbol{u} \equiv 0]=0$, it follows that $\left\langle\partial_{x} \mathscr{D}\right\rangle=0$ for any density $\mathscr{D}$. On the other hand, if $\langle\mathscr{D}\rangle=0$, then there exists some density $\Phi[\boldsymbol{u}]$ (tending to zero at infinity) such that $\mathscr{D}=\partial_{x} \Phi$, say

$$
\Phi(x, t)=-\int_{x}^{\infty} \mathscr{D}[\boldsymbol{u}(\xi, t)] \mathrm{d} \xi .
$$

Another class of solutions that will not be considered here is the class of periodic solutions.

Let us now recall the relevant concepts of velocity related to energy propagation. The energy-flux velocity, sometimes referred to as the mean velocity of energy transport, is defined as (see, e.g., [6])

$$
V_{f}:=\frac{\langle\mathscr{F}\rangle}{\langle\mathscr{E}\rangle} .
$$

The centrovelocity of energy, usually referred to simply as the centrovelocity, is defined as (see, e.g., $[9-12])$

$$
V_{\mathrm{e}}:=\lambda_{1} X_{\mathrm{e}}, \quad X_{\mathrm{e}}:=\frac{\langle x \mathscr{E}\rangle}{\langle\mathscr{E}\rangle},
$$

where $X_{\mathrm{e}}$ is the center of gravity of $\mathscr{E}$.

Of course $V_{\mathrm{f}}$ and $V_{\mathrm{e}}$ depend on the solution that is considered, i.e., $V_{\mathrm{f}}=V_{\mathrm{f}}[\boldsymbol{u}], V_{\mathrm{e}}=V_{\mathrm{e}}[\boldsymbol{u}]$ and, in general, their value will change in time for a specific solution.

The two energy velocities coincide in general only for conservative systems, as will be shown in the next section, where their interrelation will be investigated. 
Another observation to be made is that the energy velocities have a clear physical interpretation, provided that $\mathscr{E}$ has the correct physical meaning. This is important to realize, since $\mathscr{E}^{\prime \prime}=$ $\mathscr{E}+\partial_{x} \mathscr{E}^{*}$, for any density $\mathscr{E}^{*}$, has the same total energy $\langle\mathscr{E}\rangle$, but may lead to a different expression for the required energy velocity (depending on $\left.\mathscr{E}^{*}\right)$, as pointed out by Van Groesen [12] for the centrovelocity.

\section{The centrovelocity of energy}

Now we will derive for the centrovelocity $V_{\mathrm{e}}$ as defined by (2.9) an expression that we refer to as Vainshtein's formula [9]. It follows in a straightforward way by differentiating the center of gravity of $\mathscr{E}$ with respect to $t$, i.e.,

$$
\partial_{t}\left\{\left\langle\left(X_{\mathrm{e}}-x\right) \mathscr{E}\right\rangle\right\}=0,
$$

and by using the balance equation (2.3) in the general case. The differentiation leads to

$$
V_{\mathrm{e}}\langle\mathscr{E}\rangle=-X_{\mathrm{e}} \partial_{t}\langle\mathscr{E}\rangle+\left\langle x \partial_{t} \mathscr{E}\right\rangle .
$$

Integrating (2.3) over $x$ yields

$$
\partial_{\boldsymbol{t}}\langle\mathscr{E}\rangle=-\langle\mathscr{P}\rangle,
$$

and we get

$$
V_{\mathrm{e}}\langle\mathscr{E}\rangle=X_{\mathrm{e}}\langle\mathscr{P}\rangle+\langle\mathscr{F}\rangle-\langle x \mathscr{P}\rangle .
$$

Introducing the center of gravity of $\mathscr{Y}$,

$$
X_{\mathrm{s}}=\frac{\langle x \mathscr{P}\rangle}{\langle\mathscr{P}\rangle},
$$

one arrives at Vainshtein's formula:

$$
V_{\mathrm{e}}=\frac{\langle\mathscr{F}\rangle}{\langle\mathscr{E}\rangle}-\left(X_{\mathrm{s}}-X_{\mathrm{e}}\right) \frac{\langle\mathscr{T}\rangle}{\langle\mathscr{E}\rangle} .
$$

For a conserved density $\mathscr{E}(\mathscr{S}=0)$, the expression for $V_{\mathrm{e}}$ reduces to the first term of the r.h.s. of (3.5), which represents the usual energy-flux velocity $V_{f}$, as defined by $(2.8)$.

At first sight, it is rather difficult to find a clear interpretation or expression for the second term due to the loss. In the following we derive a different expression for equation (3.5) which will provide a physical interpretation of this term by means of an additional flux density $\Phi$ due to dissipation, which will be referred to as loss flux. This new approach is based on the observation that it is possible to write the loss density $\mathscr{S}$ in a unique way as

$$
\mathscr{S}=\alpha \mathscr{E}+\partial_{x} \Phi,
$$

where $\alpha=\alpha[\boldsymbol{u}(t)]$ is the functional defined by

$$
\alpha(t):=\langle\mathscr{P}\rangle /\langle\mathscr{E}\rangle,
$$

and where $\Phi$ is some density.

The proof of (3.6) follows immediately by introducing a density $\mathscr{D}$ such that

$$
\mathscr{S}=\frac{\langle\mathscr{P}\rangle}{\langle\mathscr{E}\rangle} \mathscr{E}+\mathscr{D}
$$

Then $\langle\mathscr{D}\rangle=0$, and this implies that $\mathscr{D}=\partial_{x} \Phi$ for some density $\Phi$, as stated in (2.5).

From (3.6) and (3.7) we can deduce an expression for $X_{\mathrm{s}}$ as defined by (3.4) in terms of the loss flux $\Phi$. Indeed, since

$$
\langle x \mathscr{P}\rangle=\frac{\langle\mathscr{P}\rangle}{\langle\mathscr{E}\rangle}\langle x \mathscr{E}\rangle+\left\langle x \partial_{x} \Phi\right\rangle=X_{\mathrm{e}}\langle\mathscr{P}\rangle-\langle\Phi\rangle,
$$

it follows that

$$
X_{\mathrm{s}}=X_{\mathrm{e}}-\frac{\langle\Phi\rangle}{\langle\mathscr{S}\rangle}
$$

Inserting (3.9) into (3.5) then leads to

$$
V_{\mathrm{e}}=\frac{\langle\mathscr{F}\rangle}{\langle\mathscr{E}\rangle}+\frac{\langle\Phi\rangle}{\langle\mathscr{E}\rangle}
$$

Introducing the notation

$$
V_{\phi}:=\frac{\langle\Phi\rangle}{\langle\mathscr{E}\rangle},
$$

and recalling definition (2.8), equation (3.10) reads

$$
V_{\mathrm{e}}=V_{\mathrm{f}}+V_{\phi} .
$$

Equations (3.11) and (3.12) suggest the name "loss flux" for $\Phi$. The effect of dissipation on the relation between centrovelocity and energy-flux velocity is therefore represented uniquely by this flux $\Phi$. 
The importance of the functional $\alpha$ is recognized after the following consideration. Inserting (3.6) into equation (2.3) leads to the alternative form of the energy balance, i.e.,

$$
\partial_{t} \mathscr{E}+\partial_{x}(\mathscr{F}+\Phi)=-\alpha \mathscr{E} .
$$

Integrating (3.13) over $x$ it follows that

$$
\partial_{t}\langle\mathscr{E}\rangle=-\alpha(t)\langle\mathscr{E}\rangle,
$$

from which it is found that

$$
\langle\mathscr{E}[\boldsymbol{u}]\rangle=\left\langle\mathscr{E}_{0}\right\rangle \exp \left\{-\int_{0}^{1} \alpha[\boldsymbol{u}(\tau)] \mathrm{d} \tau\right\},
$$

where $\varepsilon_{0}$ is the density corresponding to the initial data $\boldsymbol{u}(x, 0)$.

Equations (3.14) and (3.15) show that the change in $\langle\mathscr{E}\rangle$ is completely determined by $\alpha$ and that it does not depend on $\Phi$. The functional $\alpha$ is the time-attenuation factor, and the (uniquely defined) expression $\alpha \mathscr{E}$ represents the (reduced) loss density. Furthermore, from the equation (3.13) it is natural to call $(\mathscr{F}+\Phi)$ the total flux of the dissipative system.

An interesting observation about energy propagation in a nonconservative system is related to the possibility of defining an "equivalent" conservative system with respect to a rescaled energy density. In fact, with

$$
\beta[\boldsymbol{u}(t)]:=\int_{0}^{t} \alpha[\boldsymbol{u}(\tau)] \mathrm{d} \tau,
$$

the balance equation (3.13) can be rewritten as

$$
\partial_{t}\left\{\mathrm{e}^{\beta(t)} \mathscr{E}\right\}+\partial_{x}\left\{\mathrm{e}^{\beta(t)}(\mathscr{F}+\Phi)\right\}=0 .
$$

In other words, the expression

$$
\mathscr{E}^{\prime}:=\mathrm{e}^{\beta(1)} \mathscr{E}
$$

is a conserved density, with corresponding flux

$$
\mathscr{F}^{\prime}:=\mathrm{e}^{\beta(t)}(\mathscr{F}+\Phi) .
$$

It is easy to prove that the centrovelocity $V_{\mathrm{e}}^{\prime}$ corresponding to the conserved density $\mathscr{E}^{\prime}$ equals
$V_{\mathrm{e}}$. In fact, we get

$$
\begin{aligned}
V_{\mathrm{e}}^{\prime} & :=\frac{\left\langle\mathscr{F}^{\prime}\right\rangle}{\left\langle\mathscr{E}^{\prime}\right\rangle} \\
& =\frac{\left\langle\mathrm{e}^{\beta(t)}(\mathscr{F}+\Phi)\right\rangle}{\left\langle\mathrm{e}^{\beta(t) \mathscr{E}\rangle}\right.}=\frac{\langle\mathscr{F}+\Phi\rangle}{\langle\mathscr{E}\rangle},
\end{aligned}
$$

and thus, with expression (3.10) for $V_{\mathrm{e}}$,

$$
V_{\mathrm{e}}^{\prime}=V_{\mathrm{e}} \text {. }
$$

This observation shows once again the independence of the centrovelocity with respect to the attenuation factor.

When for a particular solution $\boldsymbol{u}(x, t)$ the loss density $\mathscr{S}$ is equal to the reduced loss density, i.e., $\mathscr{S}=\alpha \mathscr{E}$, then the functional $\alpha$ just represents the time-attenuation factor as defined by (3.7), and by (3.6) the corresponding loss flux vanishes. In this case we obtain for all $t$

$$
\frac{\mathscr{P}}{\mathscr{E}}=\frac{\langle\mathscr{P}\rangle}{\langle\mathscr{E}\rangle},
$$

and

$$
V_{\mathrm{e}}=V_{\mathrm{f}} .
$$

Such solutions will be referred to as uniformly damped solutions.

When we have, however, for any solution $\boldsymbol{u}(x, t)$

$$
\mathscr{P}=\alpha \mathscr{E}, \quad \alpha \text { constant, }
$$

then we refer to this case as uniform damping; then the loss flux vanishes identically and the two notions of energy-flux velocity and of centrovelocity are equivalent in spite of the presence of dissipation, as may be expected. A condition for uniform damping will be considered for linear wave equations in the next section.

\section{Energy propagation for linear wave equations}

So far there has been no need to specify the governing evolution equation: relation (3.12) between the two energy velocities (2.8) and (2.9) has been derived quite generally from the balance 
law of energy (2.3). Let us now look more specifically to this relation for a given linear wave equation in order to provide the expressions of the energy velocities associated to a solution; in particular, the flux terms $\langle\mathscr{F}\rangle,\langle\Phi\rangle$ and the attenuation factor $\alpha$ will be derived. In the Appendix we will consider two simple examples of physical interest.

We write a general linear wave equation as a system of $n$ equations of first order in time, in normal form, i.e.,

$$
\partial_{r} \boldsymbol{u}+\boldsymbol{L} \boldsymbol{u}=0
$$

where $u=u(x, t)$ is an $n$-vector function which represents the field variable and $L$ is some linear operator, acting on the spatial variable $x$.

In specific examples $L$ will be a differential or pseudo-differential operator with constant coefficients. This means that for any function $\boldsymbol{u}$ which is (spatially) Fourier transformable, the (spatial) Fourier transform of the function $v=L u$ reads

$$
\hat{v}(k)=\widehat{L u}(k):=\hat{L}(k) \hat{u}(k) .
$$

Here ${ }^{\wedge}$ denotes the transformation from $x$ to $k$ according to

$$
\begin{aligned}
& \hat{f}(k, t)=\frac{1}{\sqrt{2 \pi}} \int_{-\infty}^{+\infty} f(x, t) \mathrm{e}^{-\mathrm{i} k x} \mathrm{~d} x \\
& \Leftrightarrow f(x, t)=\frac{1}{\sqrt{2 \pi}} \int_{-\infty}^{+\infty} \hat{f}(k, t) \mathrm{e}^{+\mathrm{i} k x} \mathrm{~d} k
\end{aligned}
$$

and $\hat{L}(k)$ denotes the appropriate symbol of the (pseudo)-differential operator $L$. If, for example, $L=\partial_{x}$, then $\hat{L}(k)=\mathrm{i} k$.

Furthermore we assume that the variable $\boldsymbol{u}$ in equation (4.1) is chosen in such a way that the functional

$$
\mathscr{E}[\boldsymbol{u}]=\frac{1}{2} \boldsymbol{u} \cdot \boldsymbol{u},
$$

is the real physical energy, where - denotes the inner product in $\mathbb{R}^{n}$. This assumption is legitimate for the following reason. If the (positive definite) energy density were given by the quadratic expression

$$
\mathscr{E}[\boldsymbol{u}]=\frac{1}{2} Q \boldsymbol{u} \cdot Q \boldsymbol{u}
$$

for some nonsingular operator $Q$, then we can introduce a new variable $v=Q u$; then

$$
\mathscr{E}[\boldsymbol{v}]=\frac{1}{2} \boldsymbol{v} \cdot \boldsymbol{v},
$$

and $v$ satisfies the evolution equation

$$
\partial_{t} v+L^{\prime} v=0, \quad \text { where } L^{\prime}=Q L Q^{-1} .
$$

It should be noted that the operator $L^{\prime}$ has the same symmetry properties as $L$. In particular, the conservative character of an equation is not changed by this transformation, according to the following observation: If the system is conservative, the operator $L$ is antisymmetric, and vice versa.

Indeed, taking the $\mathbb{R}^{n}$-inner product of (4.1) with $u$, we obtain with (4.3)

$$
-\partial_{t} \mathscr{E}[u]=\boldsymbol{u} \cdot L \boldsymbol{u} .
$$

Integrating (4.7) we get

$$
-\partial_{t}\langle\mathscr{E}[\boldsymbol{u}]\rangle=\langle\boldsymbol{u} \cdot \boldsymbol{L u}\rangle=\left\langle\boldsymbol{u} \cdot L^{\mathrm{s}} \boldsymbol{u}\right\rangle,
$$

where $L^{\mathrm{s}}$ is the symmetric part of the operator $L$. Since $\left\langle\boldsymbol{u} \cdot L^{\mathrm{s}} u\right\rangle=0$ for all $\boldsymbol{u}$ if and only if (iff) $L^{\mathrm{s}}=0$, the property has been proven.

It is thus natural to call equations of the form

$$
\partial_{\imath} \boldsymbol{u}+A \boldsymbol{u}=0,
$$

conservative (with respect to the density $\mathscr{E}$ ) iff $A$ is an antisymmetric operator. For such conservative equations we have

$$
\partial_{x} \mathscr{F}=\boldsymbol{u} \cdot \boldsymbol{A} \boldsymbol{u},
$$

and we obtain the total flux as

$$
\langle\mathscr{F}\rangle=-\langle x \boldsymbol{u} \cdot A \boldsymbol{u}\rangle .
$$

In the general case we agree to write the evolution equation (4.1) in the following form

$$
\partial_{t} u+[A+S] \boldsymbol{u}=\mathbf{0},
$$

where $A$ and $S$ denote the antisymmetric and the symmetric part of the operator $L$ respectively.

Then, for nonconservative equations we get, in addition to (4.10) and (4.11),

$$
\mathscr{S}=\boldsymbol{u} \cdot S \boldsymbol{u},
$$


and

$$
\langle\mathscr{P}\rangle=\langle\boldsymbol{u} \cdot \boldsymbol{S u}\rangle .
$$

Note from (4.14) that in nonconservative systems the total loss $\langle\mathscr{P}\rangle$ may be identically zero for some particular solutions.

We are now in a position to provide, for a specific solution $\boldsymbol{u}$ of equation (4.12), the expressions of $V_{\mathrm{f}}$ and $V_{\phi}$ entering equation (3.12) which yields the corresponding centrovelocity $V_{\mathrm{e}}$.

For $V_{\mathrm{f}}$ as defined by (2.8), only the antisymmetric operator $A$ is involved to yield, using (4.3) and (4.11),

$$
V_{\mathrm{f}}=-2 \frac{\langle x \boldsymbol{u} \cdot A \boldsymbol{u}\rangle}{\langle\boldsymbol{u} \cdot \boldsymbol{u}\rangle} .
$$

For $V_{\phi}$ as defined by (3.11), we first have to derive the expression for the attenuation factor $\alpha$ as defined by (3.7), and that for the total loss flux $\langle\Phi\rangle$ as it results from (3.9). From (3.7) with (4.3) and (4.14), we get

$$
\alpha=2 \frac{\langle\boldsymbol{u} \cdot \boldsymbol{S u}\rangle}{\langle\boldsymbol{u} \cdot \boldsymbol{u}\rangle},
$$

and from (2.7), (3.4), (3.9) with (4.3), (4.14), we get

$$
\langle\Phi\rangle=\langle\boldsymbol{u} \cdot \boldsymbol{S u}\rangle\left[\frac{\langle\boldsymbol{x u} \cdot \boldsymbol{u}\rangle}{\langle\boldsymbol{u} \cdot \boldsymbol{u}\rangle}-\frac{\langle\boldsymbol{x u} \cdot S \boldsymbol{u}\rangle}{\langle\boldsymbol{u} \cdot S \boldsymbol{u}\rangle}\right] .
$$

One can use (4.16) in (4.17) to obtain the following alternative expression for $\langle\Phi\rangle$

$$
\langle\boldsymbol{\Phi}\rangle=\frac{1}{2} \alpha\langle\boldsymbol{x u} \cdot \boldsymbol{u}\rangle-\langle x \boldsymbol{u} \cdot \boldsymbol{S u}\rangle .
$$

Then, using (3.11) with (4.3) and (4.18), we get

$$
V_{\phi}=-2 \frac{\left\langle x \boldsymbol{u} \cdot\left(S-\frac{1}{2} \alpha \mathrm{I}\right) \boldsymbol{u}\right\rangle}{\langle\boldsymbol{u} \cdot \boldsymbol{u}\rangle},
$$

where I denotes the $n \times n$ identity matrix. We recognize that for $V_{\phi}$ only the symmetric part $S$ of the operator $L$ is involved.

Note that when the operator $S$ is proportional to the identity matrix, i.e.,

$$
S=\frac{1}{2} \alpha \mathrm{I} \text { for some constant } \alpha \in \mathbb{R},
$$

we find that, for any solution $u=u(x, t)$ of equation (4.11), the loss flux vanishes identically, and therefore the velocities $V_{\mathrm{f}}$ and $V_{\mathrm{e}}$ are equal.
Condition (4.20) implies, by using (4.3) and (4.13),

$$
\mathscr{S} \equiv \alpha \mathscr{E},
$$

which is the uniform damping as defined by (3.24).

In the case of uniform damping it is easy to find an "equivalent" conservative system, as defined by (3.18) and (3.19), by the following argument. Setting, in equation (4.12),

$$
\boldsymbol{u}(x, t)=\mathrm{e}^{-\alpha t / 2} v(x, t),
$$

$v$ satisfies the wave equation

$$
\partial_{\imath} v+A v=0,
$$

which is a conservative equation with respect to the energy density

$$
\mathscr{E}^{\prime}[\boldsymbol{v}]=\mathrm{e}^{\alpha t} \mathscr{E}[\boldsymbol{u}] .
$$

Furthermore, from (4.15) and (4.22) we can verify the identity of the energy velocities, i.e.,

$$
\begin{aligned}
V_{\mathrm{e}}[\boldsymbol{v}] & =-2 \frac{\langle x \boldsymbol{v} \cdot \boldsymbol{A v}\rangle}{\langle\boldsymbol{v} \cdot \boldsymbol{v}\rangle} \\
& =-2 \frac{\mathrm{e}^{-\alpha \boldsymbol{t}}\langle\boldsymbol{x u} \cdot \boldsymbol{A u}\rangle}{\mathrm{e}^{-\alpha t}\langle\boldsymbol{u} \cdot \boldsymbol{u}\rangle}=V_{\mathrm{e}}[\boldsymbol{u}] .
\end{aligned}
$$

Now we consider a particular but important class of linear wave equations which provide dispersive waves, viz. first-order hyperbolic systems (of symmetric type). We agree to write them in the form

$$
\partial_{\boldsymbol{t}} \boldsymbol{u}+D \partial_{x} \boldsymbol{u}+\boldsymbol{M u}=0,
$$

where $D$ and $M$ are constant real matrices, with $D$ symmetric, and where $\boldsymbol{u}$ is related to the energy density $\mathscr{E}$ as in (4.3). For this class, $L$ is a first-order differential operator

$$
L=D \partial_{x}+M,
$$

so that

$$
A=D \partial_{x}+M^{\mathrm{a}}, \quad S=M^{s},
$$

where $M^{\mathrm{a}}, M^{\mathrm{s}}$ denote the antisymmetric and symmetric parts of the constant matrix $M$.

For such systems we obtain particularly simple expressions for the flux $\mathscr{F}$ and for the loss $\mathscr{Y}$. From 
(4.10), (4.13) with (4.28) we get

$$
\mathscr{F}=\frac{1}{2} \boldsymbol{u} \cdot D \boldsymbol{u}, \quad \mathscr{S}=\boldsymbol{u} \cdot \boldsymbol{M}^{\mathrm{s}} \boldsymbol{u} .
$$

Consequently the energy-flux velocity and the attenuation factor read respectively

$$
V_{\mathrm{f}}=\frac{\langle\boldsymbol{u} \cdot D \boldsymbol{u}\rangle}{\langle\boldsymbol{u} \cdot \boldsymbol{u}\rangle},
$$

and

$$
\alpha=2 \frac{\left\langle\boldsymbol{u} \cdot M^{\mathrm{s}} \boldsymbol{u}\right\rangle}{\langle\boldsymbol{u} \cdot \boldsymbol{u}\rangle} .
$$

In the Appendix we will illustrate some of the foregoing for two specific examples, viz. the linear Korteweg-de Vries-Burgers (KdVB) equation and the linear Klein-Gordon equation with dissipation (KGD).

\section{Conclusions}

We have investigated the propagation of energy in dissipative systems. To that end an expression for the velocity of its center of gravity (centrovelocity) is derived from the balance law for the energy density. Such a balance law can be expressed in several equivalent ways by exchanging parts from the (pseudo-)loss to the flux term. We show that the balance law can be brought to its basic form (3.13) where the (true) loss is proportional to the energy density. In that basic form the total flux is the sum of the flux $\mathscr{F}$ of the conservative part of the system, together with the loss flux $\Phi$ which is a contribution from the dissipative part of the system. The importance for energy propagation is that this loss flux $\Phi$ enters the expression for the centrovelocity and adds to the energy-flux velocity of the conservative part of the system.

\section{Acknowledgment}

This work was partly supported by the Italian Research Council (CNR: GNFM) and by the Italian Ministry of Education (MPI: $60 \%$ grants).

\section{Appendix: The linear KdVB and KGD equations}

The linear Korteweg-de Vries-Burgers (KdVB) equation is the simplest scalar wave equation to model propagation of small-amplitude long waves in a dispersive and dissipative medium. It reads, in an obvious notation,

$$
u_{t}+c_{0} u_{x}+\mu u_{x x x}=\nu u_{x x},
$$

where $u=u(x, t)$ is the scalar field variable and $c_{0}, \mu, \nu$ are positive constants.

Defining the energy density as

$$
\mathscr{E}=\frac{1}{2} u^{2},
$$

and adopting the notation of Section 4 , we get $L=A+S$ with

$$
A=c_{0} \partial_{x}+\mu \partial_{x}^{3}, \quad S=-\nu \partial_{x}^{2} .
$$

The energy balance (2.3): $\partial_{t} \mathscr{E}+\partial_{x} \mathscr{F}=-\mathscr{P}$ is satisfied with

$$
\begin{aligned}
\mathscr{F} & =\frac{1}{2} c_{0} u^{2}+\mu\left[u u_{x x}-\frac{1}{2}\left(u_{x}\right)^{2}\right] \\
& =\frac{1}{2} c_{0} u^{2}+\frac{1}{2} \mu\left[\left(u^{2}\right)_{x x}-3\left(u_{x}\right)^{2}\right], \\
\mathscr{S} & =-\nu u u_{x x}=\nu\left[\left(u_{x}\right)^{2}-\frac{1}{2}\left(u^{2}\right)_{x x}\right] .
\end{aligned}
$$

In this way $\mathscr{F}$ is the flux of the conservative part of equation (A.1) (when $\nu=0$ ) and $\mathscr{S}$ is only related to the dissipation.

From (A.4) and (A.5) we get directly the expressions for the flux $\langle\mathscr{F}\rangle$ and the loss $\langle\mathscr{P}\rangle$ that can confirm the corresponding formulae (4.11) and (4.14) based on (A.3); we obtain

$$
\begin{aligned}
\langle\mathscr{F}\rangle & =\frac{1}{2} c_{0}\left\langle u^{2}\right\rangle-\frac{3}{2} \mu\left\langle\left(u_{x}\right)^{2}\right\rangle \\
& =-\langle x u A u\rangle \\
& =-c_{0}\left\langle x u u_{x}\right\rangle-\mu\left\langle x u u_{x x x}\right\rangle,
\end{aligned}
$$

and

$$
\langle\mathscr{P}\rangle=\nu\left\langle\left(u_{x}\right)^{2}\right\rangle=\langle u S u\rangle=-\nu\left\langle u u_{x x}\right\rangle .
$$

Now let us derive the expressions for the attenuation factor $\alpha$ and for the loss flux $\langle\Phi\rangle$ defined in (3.6) and (3.7). From (3.7), (A.2) and (A.7) we get

$$
\alpha=: \frac{\langle\mathscr{P}\rangle}{\langle\mathscr{E}\rangle}=2 \nu \frac{\left\langle\left(u_{x}\right)^{2}\right\rangle}{\left\langle u^{2}\right\rangle} \text {. }
$$


To derive the loss flux we have to decompose $\mathscr{S}$ as in (3.6), namely

$$
S=\frac{1}{2} \alpha u^{2}+\partial_{x} \Phi .
$$

This yields

$$
\begin{aligned}
& \Phi=\Phi_{1}+\Phi_{2} \\
& \text { with } \Phi_{1}=-\frac{1}{2}\left(u^{2}\right)_{x} \\
& \text { and } \partial_{x} \Phi_{2}=\nu\left(u_{x}\right)^{2}-\frac{1}{2} \alpha u^{2},
\end{aligned}
$$

so that, using (A.8),

$$
\begin{aligned}
\langle\Phi\rangle & =\left\langle\Phi_{2}\right\rangle=-\left\langle x \partial_{x} \Phi_{2}\right\rangle \\
& =-\nu\left\langle x\left[\left(u_{x}\right)^{2}-\frac{\left\langle\left(u_{x}\right)^{2}\right\rangle}{\left\langle u^{2}\right\rangle} u^{2}\right]\right\rangle .
\end{aligned}
$$

The results (A.8) and (A.11) are in agreement with the application of formulae (4.16) and (4.18) based on (A.3).

The linear Klein-Gordon equation with dissipation (KGD) is a second-order hyperbolic wave equation that reads

$$
\phi_{t t}+2 a \phi_{t}+b^{2} \phi=c^{2} \phi_{x x},
$$

where $\phi=\phi(x, t)$ and $a, b, c$ are constants.

This equation occurs in a variety of dispersive wave phenomena such as waves on a uniform stretched string that is anchored elastically to its equilibrium position by a transverse restoring force and damped by air friction. In this case $\phi$ denotes the transverse displacement and the constants $a, b, c$ are related with air damping, restoring force and string tension respectively (see, e.g., [13]).

The terms $\mathscr{E}, \mathscr{F}$ and $\mathscr{S}$ entering the energy balance are known to be respectively

$$
\begin{aligned}
& \mathscr{E}=\frac{1}{2}\left(\phi_{t}^{2}+c^{2} \phi_{x}^{2}+b^{2} \phi^{2}\right), \\
& \mathscr{F}=-c^{2} \phi_{x} \phi_{t}, \\
& \mathscr{P}=2 a \phi_{t}^{2} .
\end{aligned}
$$

The KGD equation (A.12) can be transformed into a first-order hyperbolic system which preserves the energy properties stated in (A.13-15) (see [14]).
Choosing $\boldsymbol{u}=\operatorname{col}\left(\phi_{t}, c \phi_{x}, b \phi\right)$, we obtain the required system of type (4.1) with $n=3$, where $L$ turns out to be the first-order differential operator

$$
L=D \partial_{x}+M,
$$

with

$$
\begin{aligned}
& D=\left(\begin{array}{ccc}
0 & -c & 0 \\
-c & 0 & 0 \\
0 & 0 & 0
\end{array}\right), \\
& M=\left(\begin{array}{ccc}
2 a & 0 & b \\
0 & 0 & 0 \\
-b & 0 & 0
\end{array}\right) .
\end{aligned}
$$

Also in this example we are going to derive the expressions for the attenuation factor $\alpha$ and for the loss flux $\langle\Phi\rangle$ defined in (3.6) and (3.7). From (3.7), (A.13) and (A.15) we get

$$
\alpha=: \frac{\langle\mathscr{P}\rangle}{\langle\mathscr{E}\rangle}=4 a \frac{\left\langle\phi_{t}^{2}\right\rangle}{\left\langle\left(\phi_{l}^{2}+c^{2} \phi_{x}^{2}+b^{2} \phi^{2}\right)\right\rangle} .
$$

To derive the loss flux we have to decompose $\mathscr{S}$ as in (3.6), namely

$$
\mathscr{S}=\frac{1}{2} \alpha\left(\phi_{t}^{2}+c^{2} \phi_{x}^{2}+b^{2} \phi^{2}\right)+\partial_{x} \Phi .
$$

This yields

$$
\begin{aligned}
\langle\Phi\rangle & =-\left\langle x \partial_{x} \Phi\right\rangle \\
& =-\frac{1}{2}\left\langle x\left[(4 a-\alpha) \phi_{t}^{2}-\alpha\left(b^{2} \phi^{2}+c^{2} \phi_{x}^{2}\right)\right]\right\rangle .
\end{aligned}
$$

We conclude the analysis of the two above examples with some considerations that may be of general interest.

In the examples we can note that the loss $\langle\mathscr{P}\rangle$ and consequently the attenuation factor $\alpha$ are definite positive quantities, while the loss flux $\langle\Phi\rangle$ may have either sign as can be seen by choosing appropriate initial data.

A noteworthy remark can be made on the KGD equation for which the variable $\phi$ can be expressed as

$$
\phi(x, t)=\mathrm{e}^{-a t} \psi(x, t),
$$


where $\psi$ satisfies the conservative equation

$$
\psi_{t t}+\left(b^{2}-a^{2}\right) \psi=c^{2} \psi_{x x} .
$$

The transformation (A.21) does not correspond to uniform damping since this would require a similar transformation for the complete three-vector $\boldsymbol{u}=$ $\operatorname{col}\left(\phi_{t}, c \phi_{x}, b \phi\right)$, as stated by (4.22). In our case the attenuation factor $\alpha$ clearly depends on the particular solution $\phi$ as it is seen from (A.18); we recognize that $\alpha$ can be given any value from the interval $[0,4 a]$, by choosing suitable initial data.

\section{References}

[1] T.H. Havelock, The Propagation of Disturbances in Dispersive Media, Cambridge University Press, Cambridge (1914) Chapter 6.

[2] L.J.F. Broer, "On the propagation of energy in linear conservative waves" Appl. Sci. Res. A 2, 329-344 (1951).

[3] M.A. Biot, "General theorems on the equivalence of group velocity and energy transport", Phys. Rev. 105, 1129-1137 (1957).
[4] G.B. Whitham, "Group velocity and energy propagation for three-dimensional waves", Comm. Pure Appl. Math. 14, 675-691 (1961).

[5] M.J. Lighthill, "Group velocity", J. Inst. Math. Appl. 1, 1-28 (1965)

[6] G.B. Whitham, Linear and Non Linear Waves, Wiley, New York (1974) Chapter 11.

[7] M. Hayes, "Energy flux for plane waves in linear conservative systems", J. Austral. Math. Soc. Ser. B. 20, 106-113 (1977).

[8] M. Hayes, "A note on group velocity", Proc. Roy. Soc. London. Ser. A. 354, 533-535 (1977).

[9] L.A. Vainshtein, "Group velocity of damped waves," Soviet Phys. Techn. Phys. 2, 2420-2428 (1957) (Engl. transl.).

[10] J.W. Wehausen and E.V. Laitone "Surface waves", in: S. Flügge, ed., Handbuch der Physik Vol. 9, Springer-Verlag, Berlin (1960) 446-778.

[11] R.L. Smith, "The velocities of light", Amer. J. Phys. 38, 978-984 (1970).

[12] E. van Groesen, "Unidirectional wave propagation in one-dimensional first-order Hamiltonian systems", $J$. Math. Phys. 21, 1646-1655 (1980).

[13] G.R. Baldock and T. Bridgeman, The Mathematical Theory of Wave Motion, Ellis Horwood, Chichester (1981) Chapter 5.

[14] F. Mainardi, "Energy velocity for hyperbolic dispersive waves", Wave Motion 9, 201-208 (1987). 